\title{
Ergodic directional switching in mobile insect groups
}

\author{
Carlos Escudero, ${ }^{1}$ Christian A. Yates, ${ }^{2}$ Jerome Buhl, ${ }^{3}$ Iain D. Couzin, ${ }^{4}$ Radek Erban, ${ }^{2,5}$ Ioannis G. Kevrekidis, ${ }^{6}$ and \\ Philip K. Maini ${ }^{2,7}$ \\ ${ }^{1}$ ICMAT (CSIC-UAM-UC3M-UCM), Departamento de Matemáticas, Facultad de Ciencias, Universidad Autónoma de Madrid, \\ Ciudad Universitaria de Cantoblanco, 28049 Madrid, Spain \\ ${ }^{2}$ Centre for Mathematical Biology, Mathematical Institute, University of Oxford, 24-29 St. Giles', Oxford OX1 3LB, United Kingdom \\ ${ }^{3}$ School of Biological Sciences and Centre for Mathematical Biology, Heydon-Laurence Building, A08, The University of Sydney, \\ Sydney, New South Wales, Australia \\ ${ }^{4}$ Department of Ecology and Evolutionary Biology, Princeton University, Princeton, New Jersey 08544, USA \\ ${ }^{5}$ Oxford Centre for Collaborative Applied Mathematics, Mathematical Institute, University of Oxford, 24-29 St. Giles', Oxford OX1 3LB, \\ United Kingdom \\ ${ }^{6}$ Department of Chemical Engineering, Program in Applied and Computational Mathematics and Mathematics, Princeton University, \\ Princeton, New Jersey 08544, USA \\ ${ }^{7}$ Department of Biochemistry, Oxford Centre for Integrative Systems Biology, University of Oxford, South Parks Road, Oxford OX1 3QU, \\ United Kingdom
}

(Received 7 February 2010; revised manuscript received 16 May 2010; published 29 July 2010)

\begin{abstract}
We obtain a Fokker-Planck equation describing experimental data on the collective motion of locusts. The noise is of internal origin and due to the discrete character and finite number of constituents of the swarm. The stationary probability distribution shows a rich phenomenology including nonmonotonic behavior of several order and disorder transition indicators in noise intensity. This complex behavior arises naturally as a result of the randomness in the system. Its counterintuitive character challenges standard interpretations of noise induced transitions and calls for an extension of this theory in order to capture the behavior of certain classes of biologically motivated models. Our results suggest that the collective switches of the group's direction of motion might be due to a random ergodic effect and, as such, they are inherent to group formation.
\end{abstract}

DOI: 10.1103/PhysRevE.82.011926

PACS number(s): 87.23.Cc, 05.40.-a, 05.65.+b, 87.10.Mn

\section{INTRODUCTION}

Emergence can be defined as the appearance of rich structures on a large scale resulting from a multiplicity of simple interactions at a considerably smaller scale. Collective animal motion is a paradigmatic example of such an emergent phenomenon. Depending on the species, there may exist different hierarchical levels that determine how collective displacements are realized. For example, in primate groups, an individual's dominance status can affect its role in initializing collective movement. In the case of swarming locusts, no such hierarchies are present; the ability of each individual to guide the band appears to be distributed relatively evenly throughout the insect group. Herein, we will concentrate on groups of wingless locust nymphs which form marching bands rather than flying swarms [1]. The onset of collective motion in locusts was experimentally demonstrated in [1], where it was shown that sufficiently large insect densities placed in a ring-shaped arena gave rise to a coherent displacement of the band. Low densities were characterized by random dispersal of the individuals, while for intermediate densities the coherent motion was interrupted by sudden changes of direction (hereafter referred to as "switches"). This phenomenology was partially rationalized by means of an adapted model based on that of Czirók et al. [2], who formulate a paradigmatic model for collective animal behavior in one dimension. In their original model the position, $x_{i}$, and velocity, $u_{i}$, of locust $i$ are evolved using the following two rules, identical for each individual, $i=1, \ldots, N$,

$$
x_{i}(t+1)=x_{i}(t)+v_{0} u_{i}(t),
$$

$$
u_{i}(t+1)=G\left(\bar{u}_{i}\right)+\xi_{i},
$$

where $N$ is the total number of locusts. Here $\bar{u}_{i}$ is the mean of the nondimensionalized velocities of locusts within a certain radius, $R$, of the position, $x_{i}$, of locust $i$. The function $G$ is such that $G(u)=(1+K)^{-1}[u+K \operatorname{sgn}(u)]$ for a positive constant $K$, where $\operatorname{sgn}(u)$ denotes the sign of $u$. The role of $G$ is to adjust the average nondimensionalized velocity perceived by each particle toward unity. $v_{0}$ is a constant associated with the chosen time scale and $\xi_{i}$ is a random number drawn from the uniform distribution in $[-\eta / 2, \eta / 2]$. The adapted version of the model used in [1] to model the movements of locust nymphs in a quasi-one-dimensional arena takes the form

$$
\frac{d x_{i}}{d t}=u_{i}, \quad d u_{i}=\left[G\left(\bar{u}_{i}\right)-u_{i}\right] d t+\beta_{1} d W_{i}, \quad \text { for } i=1, \ldots, N,
$$

where $d W_{i}$ denotes the increments of independent Wiener processes, $\beta_{1}$ is a positive constant describing the amplitude of the noise and the function $G$ is as above.

A biologically motivated refinement of the model described by Eq. (1) was given in [3], where it was postulated that individual locusts increase the randomness of their movements in response to a loss of group alignment. This behavior is the result of a particular multiplicative form of the noise term [see Eq. (2)], as opposed to the additive noise in Eq. (1); this characteristic was shown to increase the coherence of the group motion and to reduce the frequency of direction switches [3]. The key point in the analysis per- 
formed in [3] was the estimation of coefficients of an effective Fokker-Planck equation (FPE) [4], which is written in terms of a macroscopic (low-dimensional) observable [5], the average velocity of the marching group, derived directly from the experimental data. In the present work, we approximate the drift and diffusion coefficients of the effective FPE by analytical functions. This permits a more thorough analysis and fosters further understanding of collective dynamics of locusts. In addition we compare our results with those of Eq. (1), and discuss the disparities between the two models.

\section{MODEL}

Coarse-grained analysis [4] allows us to obtain an effective FPE describing the collective behavior of the locusts at the macroscopic level. By using this coarse-graining technique (see [3]) we were able to extract the coefficients of the assumed underlying FPE describing the alignment of the locusts from the experimental data presented in [1]. This approach enables us to reduce our system-comprising a large number of degrees of freedom-to a single collective variable, $u$, (referred to variably, hereafter, as "alignment" or "average velocity") which characterizes the system's macroscopic behavior. The proposed FPE has a simple form and it can be expressed as

$$
\partial_{t} P=-\alpha_{2} \partial_{u}\left[\left(u-\frac{u^{3}}{1-u^{2}}\right) P\right]+\frac{\beta_{2}}{N} \partial_{u u}\left[\left(1-u^{2}\right) P\right],
$$

for the probability $P(u, t) d u d t$ of finding the system with an average velocity in the interval $(u, u+d u)$ during the time interval $(t, t+d t)$; note that the experimental situation in [1] is quasi-one-dimensional, allowing the use of a onedimensional FPE [3]. We note that this FPE corresponds to the following Langevin equation for the average velocity

$$
d u=\alpha_{2}\left(u-\frac{u^{3}}{1-u^{2}}\right) d t+\sqrt{\frac{2 \beta_{2}}{N}} \sqrt{1-u^{2}} d W,
$$

where $d W$ denotes the increments of a Wiener process and the multiplicative noise is interpreted in the Itô sense, as prescribed by the experimentally obtained FPE (2). In these equations the average velocity $u$ is dimensionless and takes its values in the interval $[-1,1]$. The values such that $|u|$ $=1$ characterize the ideal situation in which all locusts march in perfect coherence; the sign determines the direction. Of course, formally substituting $|u|=1$ in Eq. (3) produces a divergence in the drift, so for practical reasons one has to assume that coherent motion implies $|u| \lesssim 1$ rather than a strict equality. The value $u=0$ characterizes a total disorder; realistic values of the average velocity lie between these two extreme cases. Although the average velocity is a dimensionless quantity in Eqs. (2) and (3), time is not. Consequently $\alpha_{2}$ and $\beta_{2}$ have the dimensions of time ${ }^{-1}$. We shall estimate in the following their numerical values using the experimental data from [1] and express them in units of seconds ${ }^{-1}$.

The proposed FPE (2) describing the alignment is a reasonably accurate approximation to the unknown FPE assumed to underly the motion of the locusts, which captures their experimental swarming behavior. It should be noted that such an equation can only be obtained if the system being studied is amenable to this sort of reduction.

For asymptotically large values of $N$, parameter $\alpha_{2}^{-1}$ [in Eq. (2)] denotes the order of magnitude of the relaxation time characterizing how long it takes the entire group to become ordered when starting from a disordered configuration, and $N \beta_{2}^{-1}$ indicates the order of magnitude of the characteristic time over which the fluctuations of the mean velocity develop. For the range of experimentally considered locust numbers $(5 \leq N \leq 40)$ the observed values of $\alpha_{2}$ and $\beta_{2}$ are approximately constant while we expect the presence of a boundary layer for smaller values of $N$. Since our results in [3] are rather noisy our goal is to fit the order of magnitude of the model parameters instead of attempting to obtain precise estimates. Comparing the proposed analytical coefficients of Eq. (2) to those obtained in [3], from the experimental data in [1], we obtain $\beta_{2} / \alpha_{2}=2.4 \pm 1.7$. Employing the mean switching time measurements in [3] we find $\alpha_{2}$ $=(6.65 \pm 2.63) 10^{-4} \mathrm{~s}^{-1}$ and $\beta_{2}=(1.62 \pm 0.52) 10^{-3} \mathrm{~s}^{-1}$.

The FPE corresponding to Eq. (1) can be obtained as a mean-field approximation,

$$
\partial_{t} P=-\alpha_{1} \partial_{u}\{[\operatorname{sgn}(u)-u] P\}+\frac{\beta_{1}}{N} \partial_{u u} P,
$$

where $\alpha_{1}=K /(1+K)$ and $K$ is defined as for the function $G$ in Eq. (1). The stationary solution of the FPE (4) can be derived as follows:

$$
P_{s}(u)=\frac{\sqrt{\frac{\alpha_{1} N}{2 \pi \beta_{1}}} \exp \left(-\frac{\alpha_{1} N}{2 \beta_{1}}\right)}{1+\operatorname{erf}\left(\sqrt{\frac{\alpha_{1} N}{2 \beta_{1}}}\right)} \exp \left[\frac{\alpha_{1} N}{\beta_{1}}\left(|u|-\frac{1}{2} u^{2}\right)\right] .
$$

The values of the two maxima of this stationary probability distribution (SPD) $u_{\max }= \pm 1$ and the minimum $u_{\min }=0$ are independent of the parameter values. This type of system has been considered many times in the literature [6,7], and we include it here simply for completeness and comparison with the refined model Eq. (3).

In the absence of sources and sinks of probability, we can also derive the SPD of the experimentally motivated FPE (2):

$$
P_{s}(u)=\mathcal{N}\left(1-u^{2}\right)^{-1-N \alpha_{2} / \beta_{2}} \exp \left[-\frac{N \alpha_{2} /\left(2 \beta_{2}\right)}{1-u^{2}}\right],
$$

where $\mathcal{N}^{-1}=\int_{-1}^{1}\left(1-u^{2}\right)^{-1-N \alpha_{2} / \beta_{2}} \exp \left\{-N \alpha_{2} /\left[2 \beta_{2}\left(1-u^{2}\right)\right]\right\} d u$ is the inverse of the normalization constant. This SPD is bounded, compactly supported in $[-1,1]$ and bimodal for all values of the parameters.

Noise induced transitions have been studied traditionally by means of the dynamics of the extrema of the SPD [8]. For the biologically motivated FPE (2) the SPD shows one minimum always located at $u_{\min }=0$, and two maxima at $u_{\max }$ $= \pm \sqrt{\alpha_{2}+2 \beta_{2} / N} / \sqrt{2 \alpha_{2}+2 \beta_{2} / N}$, which exist for all parameter values. One immediately notes $\left|u_{\max }\right| \in(1 / \sqrt{2}, 1)$, a fact 
related to the shape of the "deterministic potential" (the potential in the absence of noise), which is the negative integral of the drift coefficient,

$$
\mathcal{V}(u)=-\int_{0}^{u} \alpha_{2}\left(s-\frac{s^{3}}{1-s^{2}}\right) d s=-\alpha_{2}\left[u^{2}+\frac{1}{2} \ln \left(1-u^{2}\right)\right] .
$$

This potential is bistable with one maximum located at the origin and two minima at $\pm 1 / \sqrt{2}$ independent of the parameter values. For increasing noise intensity the probability maxima of the SPD Eq. (6) [corresponding to the biologically motivated FPE (2)] separate from the deterministic potential minima $\pm 1 / \sqrt{2}$ and approach the boundary points \pm 1 . These facets of the SPD, when considered in the context of the classical theory of noise induced transitions, imply that the system is becoming ordered [8]: the SPD maxima, representing the states in which the system will most likely be found, are further apart and thus there is a clearer differentiation between those states. However, the experimental evidence, based on switching times which decrease as the noise magnitude increases, reveals that the system becomes disordered [1]. This indicates that for complex systems, restricting the characterization of the dynamics to observations of the evolution of the extrema may not be adequate in some experimentally motivated situations. Herein we will try to carry out a more complete characterization.

\section{BARRIER HEIGHT}

Another indicator of order and/or disorder is the barrier height of the effective potential. For the model given by Eq. (1) the barrier height decreases monotonically as the noise intensity increases as can be seen from Eq. (8),

$$
\begin{gathered}
\mathcal{V}_{\text {eff }}(u)=-\frac{\alpha_{1} N}{\beta_{1}}\left(|u|-\frac{1}{2} u^{2}\right), \\
\Delta \mathcal{V}_{\text {eff }} \equiv \mathcal{V}_{\text {eff }}\left(u_{\text {min }}\right)-\mathcal{V}_{\text {eff }}\left(u_{\text {max }}\right)=\frac{\alpha_{1} N}{2 \beta_{1}},
\end{gathered}
$$

where $\mathcal{V}_{\text {eff }}$ is the effective potential and $\Delta \mathcal{V}_{\text {eff }}$ the corresponding barrier height.

The effective potential for our revised model [Eq. (2)] is given as

$$
\mathcal{V}_{\mathrm{eff}}(u) \equiv-\ln \left[P_{s}(u)\right]=\frac{N \alpha_{2}}{2 \beta_{2}\left(1-u^{2}\right)}+\left(1+\frac{N \alpha_{2}}{2 \beta_{2}}\right) \ln \left(1-u^{2}\right),
$$

and the corresponding barrier height is

$$
\begin{aligned}
\Delta \mathcal{V}_{\text {eff }} & \equiv \mathcal{V}_{\text {eff }}\left(u_{\text {min }}\right)-\mathcal{V}_{\text {eff }}\left(u_{\text {max }}\right) \\
& =-1-\frac{N \alpha_{2}}{2 \beta_{2}}+\left(1+\frac{N \alpha_{2}}{\beta_{2}}\right) \ln \left(2+\frac{2 \beta_{2}}{N \alpha_{2}}\right) .
\end{aligned}
$$

As a function of noise intensity the barrier height exhibits a minimum at $\left[\beta_{2} /\left(N \alpha_{2}\right)\right]_{\min } \approx 0.76$. This means that for $\beta_{2} /\left(N \alpha_{2}\right)<\left[\beta_{2} /\left(N \alpha_{2}\right)\right]_{\min }$ (subthreshold) the barrier height

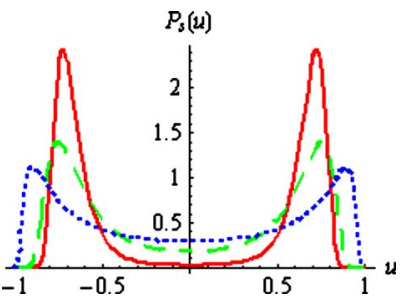

(a)

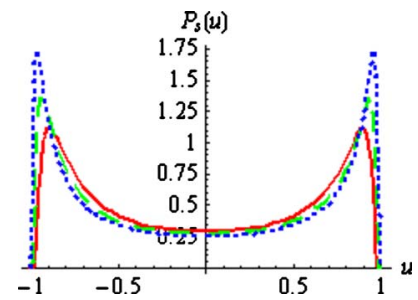

(b)

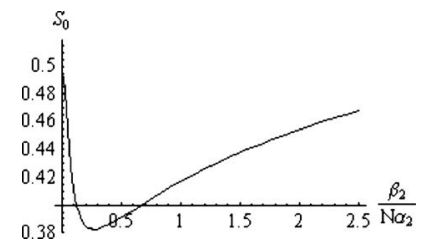

(c)

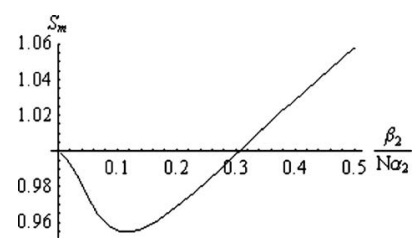

(d)

FIG. 1. (Color online) Panels (a) and (b) show the profile of the $\mathrm{SPD}, P_{s}(u)$, from Eq. (6) plotted against the normalized mean velocity $u$ for a varying noise intensity $\beta_{2} /\left(N \alpha_{2}\right)$. In panel (a) the solid red line represents $\beta_{2} /\left(N \alpha_{2}\right)=0.05$, the dashed green line represents $\beta_{2} /\left(N \alpha_{2}\right)=0.15$ and the dotted blue line represents $\beta_{2} /\left(N \alpha_{2}\right)=1.5$. In panel (b) the solid red line represents $\beta_{2} /\left(N \alpha_{2}\right)$ $=1.5$, the dashed green line represents $\beta_{2} /\left(N \alpha_{2}\right)=3.0$ and the dotted blue line represents $\beta_{2} /\left(N \alpha_{2}\right)=5.5$. Panel (c) displays the second moment of the revised model, centered at the origin, $S_{0}$, versus noise strength $\beta_{2} /\left(N \alpha_{2}\right)$ and panel (d) shows the second moment at a maximum, $S_{m}$, versus noise strength $\beta_{2} /\left(N \alpha_{2}\right)$. The minima are attained for $\beta_{2} /\left(N \alpha_{2}\right) \approx 0.27\left(S_{0}\right)$ and for $\beta_{2} /\left(N \alpha_{2}\right) \approx 0.12\left(S_{m}\right)$.

diminishes for stronger noise, but for $\beta_{2} /\left(N \alpha_{2}\right)$ $>\left[\beta_{2} /\left(N \alpha_{2}\right)\right]_{\text {min }}$ (superthreshold) it increases as the noise strength grows. Indeed, $\Delta \mathcal{V}_{\text {eff }} \approx[\ln (2)-1 / 2]\left(N \alpha_{2} / \beta_{2}\right)$ when $N \alpha_{2} / \beta_{2} \rightarrow \infty$ and $\Delta \mathcal{V}_{\text {eff }} \approx-\ln \left(N \alpha_{2} / \beta_{2}\right)$ when $N \alpha_{2} / \beta_{2} \rightarrow 0$. This suggests that, while increased noise causes the system to become more disordered for subthreshold noise intensities, superthreshold intensities might cause the system to become more ordered as the noise grows. In short, the "barrier height" order parameter shows a clear nonmonotonicity when considered as a function of noise strength. This appears like a counterintuitive reentrant behavior, where the noise can have an ordering effect for supercritical intensities [8]. Although this behavior is interesting in itself, it is not biologically relevant, as it requires a number of individuals $N$ $\approx 3$, beyond the validity of the model. Both characteristics of the SPD Eq. (6) of our refined model, displacement of the location of the maxima and nonmonotonic variation of the barrier height, can be seen in Figs. 1(a) and 1(b).

Let us note that noise induced nonequilibrium phase transitions $[9,10]$ as well as other noise mediated ordering phenomena [11] have been exhaustively studied in the literature. This includes the detailed study of reentrant transitions [12]. However, most of these approaches have assumed multiplicative noise interpreted in the Stratonovich sense. The Stratonovich interpretation is able to destabilize a deterministically stable state and in this way produce phase transitions or other noise induced phenomena. The key technical point in these cases is the appearance of a systematic contribution to the deterministic dynamics coming from the noise term, the so-called "Stratonovich drift" [8]. Therefore, noise interpre- 
tation plays a fundamental role in the development of these types of phenomena: in particular, many noise induced phenomena are not possible if the noise interpretation is that of Itô. In this respect, our results are fundamentally different as we only consider the Itô interpretation for our Langevin equation with multiplicative noise Eq. (3). We also note that noise induced phase transitions which are independent of the noise interpretation have also been studied [13], but in much less detail. The mechanisms leading to these phase transitions are based on the bifurcation of the minima of an effective potential due to noise and, as such, constitute the natural extension of noise induced transitions [8] to spatially extended systems. Our results relate to a zero dimensional system as do those in [8], but they are significantly different as the model defined by Eq. (3) does not describe this kind of bifurcation.

\section{MEAN SWITCHING TIME}

We can further explore the model properties by considering the mean switching time, $T(u)$, defined as the first time, on average, that the alignment of the system, $u$, initialized such that $-1<u<0$, reaches the origin $(u=0)$. For our revised model the moments of the switching time distribution are given, recursively, by the solution of the equation

$$
\alpha_{2}\left(u-\frac{u^{3}}{1-u^{2}}\right) \partial_{u} T_{n}+\frac{\beta_{2}}{N}\left(1-u^{2}\right) \partial_{u u} T_{n}=-n T_{n-1},
$$

subject to the boundary conditions $T(0)=0$ and $T^{\prime}(-1)=0$, where $T_{n}$ is the $n$th moment, correspondingly, $T \equiv T_{1}$ is the mean switching time and $T_{0} \equiv 1$. The second boundary condition represents zero probability flux through $u=-1$. Equation (11) is directly derived from the FPE using methods from [14]. The solution to this equation for $n=1$ is

$$
\begin{aligned}
T(u)= & \frac{N}{\beta_{2}} \int_{u}^{0} \exp \left[\frac{N \alpha_{2} /\left(2 \beta_{2}\right)}{1-v^{2}}\right]\left(1-v^{2}\right)^{N \alpha_{2} / \beta_{2}} \\
& \times \int_{-1}^{v} \exp \left[-\frac{N \alpha_{2} /\left(2 \beta_{2}\right)}{1-w^{2}}\right]\left(1-w^{2}\right)^{-1-N \alpha_{2} / \beta_{2}} d w d v .
\end{aligned}
$$

This expression appears complicated, but one can derive its asymptotic expansion for large values of $N \alpha_{2} / \beta_{2}$ (which implies large $N$ as $\alpha_{2}$ and $\beta_{2}$ are approximately constant) by means of a Kramers-like approximation [15]. It has the simple form $T(-1 / \sqrt{2}) \approx \frac{\sqrt{2} \pi}{\alpha_{2}}\left(\frac{2}{\sqrt{e}}\right)^{N \alpha_{2} / \beta_{2}}$, which reveals a pure exponential growth in the inverse noise intensity $N \alpha_{2} / \beta_{2}$ for asymptotically large values. Further moments of the switching time distribution can be calculated from Eq. (11) for $n$ $>1$. In the limit $N \rightarrow \infty$ one finds the relation $T_{n}=n ! T^{n}$. This relationship implies, in turn, that the switching process is a Poisson process.

We can also compute the first passage time for the model Eq. (1). In this case, we solve the equation $\alpha_{1}[\operatorname{sgn}(u)$ $-u] \partial_{u} T+\frac{\beta_{1}}{N} \partial_{u u} T=-1$, subject to the boundary conditions $T(0)=0$ and $T^{\prime}(-\infty)=0$, where the latter condition is the ana$\log$ of the previous zero flux condition adapted for an SPD with infinite support. We find

$$
\begin{aligned}
T(u)= & \sqrt{\frac{\pi N}{2 \alpha_{1} \beta_{1}}} \int_{u}^{0} \exp \left[\frac{N \alpha_{1}}{2 \beta_{1}}(1+v)^{2}\right] \\
& \times\left\{2-\operatorname{erfc}\left[\sqrt{\frac{\alpha_{1} N}{2 \beta_{1}}}(1+v)\right]\right\} d v,
\end{aligned}
$$

which also behaves exponentially in $N$ for large values of $N$ but this time with an $N$ dependent prefactor (see Supplementary Information of [3]). The relation between these two mean switching times [the model Eq. (1) and that of the revised model [3]] is extensively discussed in [3], so we will not reproduce the discussion here.

Now we compare the theoretical results with the exponential fitting we have performed on experimental data from [1] for both the first and second moments of the switching time distribution. The data are insufficient for us to reliably obtain any moments higher than the second. For the mean switching time $T$ and second moment $T_{2}$ we found

$$
\begin{gathered}
T=(970 \pm 120) \exp [(0.045 \pm 0.007) N] \mathrm{s}, \\
\sqrt{T_{2} / 2}=(1300 \pm 190) \exp [(0.041 \pm 0.008) N] \mathrm{s} .
\end{gathered}
$$

According to the relation $T_{n}=n ! T^{n}$ for the moments of the exponential distribution, these two values should be the same if the switching process were Poissonian. Note that the exponential growth is the same for both (within errors), while the prefactor is larger for the second moment. This suggests that the switching process is Poissonian for large $N$, that is, the probability distribution for the switching events is $\mathcal{P}$ $=T^{-1} \exp (-t / T)$. For small values of $N$ the behavior is more stochastic, as signaled by the larger prefactor of the second moment (when $N$ is small the prefactor dominates over the exponential). If the switching process is Poissonian then this has a series of consequences concerning predictability: the standard deviation being equal to the mean implies a $100 \%$ error in predictions. Furthermore, switching events are uncorrelated and the distribution tail falls off exponentially for long times. This allows for a higher probability of rare events than would be allowed by a Gaussian tail. This also implies that the switching process is Markovian, as predicted by the FPE. This can be seen from the double-welled FPE (2) in the large $N$ limit. After a short time the system relaxes to one potential minimum where it stays an exponentially long time until the switch occurs. Since practically all switches start at the minimum this erases the memory and the Markov property is recovered. The verification of this theoretical prediction by the experimental data suggests that no important correlations have been suppressed in the coarse-grained computation in [3], and that this method, and the FPE (2), are suitable to describe the locust dynamics exhibited by the experimental data.

\section{SECOND MOMENTS}

Another indicator of the stochastic properties of the system is the second moment, which measures the spread of the mean velocity, $u$, with respect to some reference value. We consider two variants, one centered at the origin $S_{0}$ 
$\equiv \int_{-1}^{1} u^{2} P_{s}(u) d u$, and one centered at one of the maxima of the probability distribution $S_{m} \equiv \int_{-1}^{1}\left(u-u_{\max }\right)^{2} P_{s}(u) d u$. Of course, the value of $S_{m}$ is the same for both maxima as a consequence of the symmetry of the system. These integrals have been computed numerically and are represented in Fig. 1(c), centered at the origin, and Fig. 1(d) centered at a maximum. Both show nonmonotonic behavior in noise intensity, but attain their minima for different values of the noise amplitude. This nonmonotonic behavior, as well as the behavior of the effective barrier height, are not reflected in the relationship between mean switching time and the size of the noise parameter $\left(N \alpha_{2} / \beta_{2}\right)$ : the mean switching time grows monotonically with noise amplitude. For comparison we note that both moments $S_{0}$ and $S_{m}$ grow monotonically with the inverse noise intensity in the model given by Eq. (1); in this case they are

$$
\begin{gathered}
S_{0}=1+\frac{\beta_{1}}{\alpha_{1} N}+\frac{\sqrt{\frac{2 \beta_{1}}{\pi \alpha_{1} N}} \exp \left(-\frac{\alpha_{1} N}{2 \beta_{1}}\right)}{1+\operatorname{erf}\left(\sqrt{\left.\frac{\alpha_{1} N}{2 \beta_{1}}\right)},\right.} \\
S_{m}=2+\frac{\beta_{1}}{\alpha_{1} N}+\frac{\sqrt{\frac{2 \beta_{1}}{\pi \alpha_{1} N}} \exp \left(-\frac{\alpha_{1} N}{2 \beta_{1}}\right)}{1+\operatorname{erf}\left(\sqrt{\frac{\alpha_{1} N}{2 \beta_{1}}}\right)} .
\end{gathered}
$$

There is another feature of the second moments of the revised model [3], in addition to the nonmonotonic behavior, that reveals new characteristics of the collective motion of locusts not reflected by the model Eq. (1). In this model, a reduction in the number of individuals increases the values of both second moments. In the stronger noise situation the probability distribution tails grow, which implies that there are more individuals with a higher (absolute value) velocity. In our case the probability is compactly supported in $[-1,1]$, as a consequence of the biological fact that the propagation cannot be better than perfect. For realistic values of the parameters the system is in the weak noise regime [see Fig. 1(c)]. This means that the second moment centered at the origin decreases for a decreasing number of locusts, exactly the opposite trend to that of the model Eq. (1). The reason is that the probability of finding the system in the neighborhood of $u=0$ grows considerably for stronger noise (as reflected by the decreasing barrier height), largely compensating for the drift of the maxima toward the boundaries of the support of the SPD. The experimentally derived value of $\beta_{2} / N \alpha_{2}=0.12 \pm 0.08$ for $N=20$ agrees with the minimizing value of the second moment centered at a maximum, $\beta_{2} / N \alpha_{2} \approx 0.12$ [see Fig. $1(\mathrm{~d})$ ]. This implies that its behavior is not very sensitive to small changes in the number of locusts.

\section{CONCLUSIONS}

We have seen that the FPE obtained from the coarsegrained analysis of experimental data on the movement of locusts shows an interesting phenomenology. Different indicators of order and/or disorder may vary nonmonotonically with noise intensity, possibly in a contradictory manner. These findings reveal that these indicators might not be suitable for the biologically motivated models studied in this paper. We have also shown that the direction switches are independently distributed for large numbers of individuals. This makes them almost unpredictable from a practical viewpoint. It seems that directional switches are produced by an accumulation of errors (made by the locusts when trying to adapt their velocity to that of their neighbors) that ordinarily interfere and cancel each other out but, over exponentially long times, have the possibility of accumulating and producing a switch. According to the results presented here, specifically the confirmation of the Poissonian character of the switching events, it seems possible that directional switches are produced as a consequence of the ergodic random evolution of the system. We note the similarity of this process with Ising model ergodic magnetization changes [16]. Indeed, the model of Eq. (1) can be thought of as an Ising model with moving spins. It seems that the ergodic nature of the finite size Ising model is preserved despite introducing movement of the spins. More importantly it seems that this is a plausible explanation, in the absence of external stimuli, of the sudden changes of direction observed in animal groups.

\section{ACKNOWLEDGMENTS}

The authors are grateful to David Sumpter for useful comments and discussions. This work was supported by the Oxford-Princeton Research Partnership grant. C.E. acknowledges support by the MICINN (Spain) through Project No. MTM2008-03754. C.A.Y. thanks EPSRC for funding via the Systems Biology Doctoral Training Centre, University of Oxford. J.B. was funded by the Australian Research Council (ARC) Linkage and Discovery programs. I.D.C. acknowledges support from the Searle Scholars Program (Grant No. 08-SPP-201), National Science Foundation (Grant No. PHY0848755), Office of Naval Research (Grant No. N00014-091-1074) and a DARPA Grant (Grant No. HR0011-05-10057). This publication was based on work (R.E.) supported in part by Award No. KUK-C1-013-04, made by King Abdullah University of Science and Technology (KAUST); R.E. also thanks Somerville College, Oxford for support. The research leading to these results has received funding from the European Research Council under the European Community's Seventh Framework Programme (FP7/2007-2013)/ERC Grant Agreement No. 239870. I.G.K. was partially supported by the AFOSR. P.K.M. was partially supported by the Royal Society. 
[1] J. Buhl, D. J. T. Sumpter, I. D. Couzin, J. J. Hale, E. Despland, E. R. Miller, and S. J. Simpson, Science 312, 1402 (2006).

[2] A. Czirók, A.-L. Barabási, and T. Vicsek, Phys. Rev. Lett. 82, 209 (1999).

[3] C. A. Yates, R. Erban, C. Escudero, I. D. Couzin, J. Buhl, I. G. Kevrekidis, P. K. Maini, and D. J. T. Sumpter, Proc. Natl. Acad. Sci. U.S.A. 106, 5464 (2009).

[4] R. Erban, I. G. Kevrekidis, D. Adalsteinsson, and T. C. Elston, J. Chem. Phys. 124, 084106 (2006).

[5] R. Erban, T. A. Frewen, X. Wang, T. C. Elston, R. Coifman, B. Nadler, and I. G. Kevrekidis, J. Chem. Phys. 126, 155103 (2007).

[6] P. Hänggi, P. Talkner, and M. Borkovec, Rev. Mod. Phys. 62, 251 (1990).

[7] L. Gammaitoni, P. Hänggi, P. Jung, and F. Marchesoni, Rev. Mod. Phys. 70, 223 (1998).

[8] W. Horsthemke and R. Lefever, Noise-Induced Transitions
(Springer-Verlag, Berlin, 1984).

[9] C. Van den Broeck, J. M. R. Parrondo, and R. Toral, Phys. Rev. Lett. 73, 3395 (1994).

[10] C. Van den Broeck, J. M. R. Parrondo, R. Toral, and R. Kawai, Phys. Rev. E 55, 4084 (1997).

[11] J. García-Ojalvo and J. M. Sancho, Noise in Spatially Extended Systems (Springer, New York, 1999).

[12] R. Kawai, X. Sailer, L. Schimansky-Geier, and C. Van den Broeck, Phys. Rev. E 69, 051104 (2004).

[13] O. Carrillo, M. Ibañes, J. García-Ojalvo, J. Casademunt, and J. M. Sancho, Phys. Rev. E 67, 046110 (2003).

[14] C. W. Gardiner, Handbook of Stochastic Methods (SpringerVerlag, Berlin, 1996).

[15] C. Escudero and A. Kamenev, Phys. Rev. E 79, 041149 (2009).

[16] K. Brendel, G. T. Barkema, and H. van Beijeren, Phys. Rev. E 67, 026119 (2003). 